\title{
Scattering lengths in $S U(2)$ gauge theory with two fundamental fermions
}

\author{
R. Arthur ${ }^{a}$, V. Drach ${ }^{* a}$, M. Hansen ${ }^{a}$, A. Hietanen ${ }^{a}$, C. Pica ${ }^{a}$, F. Sannino ${ }^{a}$ \\ ${ }^{a} C P^{3}$-Origins \& the Danish Institute for Advanced Study DIAS, University of Southern Denmark, \\ Campusvej 55, DK-5230 Odense M, Denmark \\ E-mail: drach@ep3.dias.sdu.dk
}

\begin{abstract}
We investigate non perturbatively scattering properties of Goldstone Bosons in an $S U(2)$ gauge theory with two Wilson fermions in the fundamental representation. Such a theory can be used to build extensions of the Standard Model that unifies Technicolor and pseudo Goldstone composite Higgs models. The leading order contribution to the scattering amplitude of Goldstone bosons at low energy is given by the scattering lengths. In the context of technicolor extensions of the Standard Model the scattering lengths are constrained by WW scattering measurements. We first describe our setup and in particular the expected chiral symmetry breaking pattern. We then discuss how to compute them on the lattice and give preliminary results using finite size methods.
\end{abstract}

The 32nd International Symposium on Lattice Field Theory,

23-28 June, 2014

Columbia University New York, $N Y$

${ }^{*}$ Speaker. 


\section{Introduction}

In this work we consider an $S U(2)$ gauge field theory with two fermions in the fundamental representation. The Lagrangian reads in the continuum :

$$
\mathscr{L}=-\frac{1}{4} F_{\mu \nu}^{a} F^{a \mu \nu}+\bar{\psi}(i \not D-m) \psi,
$$

where $\psi=(u, d)$ is a doublet of Dirac spinor fields transforming according to the fundamental representation.

Because of the pseudo-realness of the fundamental representation of $S U(2)$, the mass term of the Lagrangian can be written in terms of 4 Weyl spinors as follows ${ }^{1}$

$$
\left.\mathscr{L}=-\frac{1}{4} F_{\mu \nu}^{a} F^{a \mu v}+\bar{\psi} i \not D \psi+\frac{i m}{2} Q^{T}\left(-i \sigma_{2}\right) C E Q+\left(Q^{T}\left(-i \sigma_{2}\right) C E Q\right)\right)^{\dagger}
$$

where $\sigma_{2}$ acts on color indices and $C$ is the charge conjugation matrix. Furthermore, we have defined :

$$
Q=\left(\begin{array}{c}
u_{L} \\
d_{L} \\
-i \sigma_{2} C \bar{u}_{R}^{T} \\
-i \sigma_{2} C \bar{d}_{R}^{T}
\end{array}\right) \text {, and } E=\left(\begin{array}{cccc}
0 & 0 & +1 & 0 \\
0 & 0 & 0 & +1 \\
-1 & 0 & 0 & 0 \\
0 & -1 & 0 & 0
\end{array}\right)
$$

We have used $q_{L, R}=P_{L, R} q, \bar{q}_{L, R}=\bar{q} P_{R, L}$ with $P_{L}=\frac{1}{2}\left(1-\gamma_{5}\right)$ and $P_{R}=\frac{1}{2}\left(1+\gamma_{5}\right)$. The model exhibits an $S U(4)$ flavour symmetry in the massless limit. To fix notations, the 15 generators of the corresponding Lie algebra will be denoted $T^{a=1, \ldots, 15}$. After adding a mass term, the remnant flavour symmetry is the group spanned by the algebra that preserves $E T^{a, T}+T^{a, T} E=0$. This relation defines the 10-dimensional algebra of the $S P(4)$ group. The chiral symmetry breaking pattern is thus expected to be $S U(4) \rightarrow S P(4)$ leading to 5 Goldstone bosons.

As proposed in [2], the Lagrangian Eq. (1.1) can be embedded into the Standard Model in such a way that it interpolates between composite Goldstone Higgs and Technicolor models [3, 4].

The model has been investigated on the lattice in [5], and the chiral symmetry breaking pattern has been proven to be the expected one [6]. Updated results concerning our on-going effort are summarized in [7].

Since one feature of the model is to provide a dynamical explanation of Electroweak symmetry breaking, calculating the scattering properties of the Goldstone bosons of the underlying theory can be related to scattering properties of longitudinal $\mathrm{W}$ bosons according to the equivalence theorem[8].

The two particle states involving two Goldstone bosons can be classified according $\mathbf{5} \otimes \mathbf{5}=$ $\mathbf{1} \oplus \mathbf{1 0} \oplus \mathbf{1 4}$ and it can be shown that $\pi^{+} \pi^{+}$belongs to the $\mathbf{1 4}$ dimensional representation of $\operatorname{SP}(4)$.

The low energy prediction for this realization of chiral symmetry breaking has been considered in[9], and reads:

$$
m_{\mathrm{PS}} a_{0, L O}^{\mathbf{1 4}}=-\frac{m_{\mathrm{PS}}^{2}}{32 f_{\mathrm{PS}}^{2}}
$$

\footnotetext{
${ }^{1}$ In fact $S U(2)$ is the first of the $S p(2 N)$ gauge theories. The associated conformal window was studied in [1].
} 
where $m_{\mathrm{PS}}$ and $f_{\mathrm{PS}}$ are respectively the mass and decay constant of the Goldstone bosons, and where $a_{0, L O}^{\mathbf{1 4}}$ is the scattering length related to the partial wave amplitude at zero-momentum transfer of the scattering process : $\pi^{+} \pi^{+} \rightarrow \pi^{+} \pi^{+}$. The superscript 14 refers to the dimension of the corresponding irreducible representation of $S P(4)$.

\section{Lattice techniques}

In order to obtain the scattering lengths from lattice simulations in Euclidean space, we used the same strategy as in QCD to compute scattering lengths of pions in the isospin 2 channel (see e.g [10]). We briefly review here our approach.

The general idea is to compute the energy of the two Goldstone bosons in a finite box. Lüscher's finite size method [11], relates the energy shift due to the interactions of the two particles in a finite box to their infinite volume scattering length according to the following formula:

$$
\frac{\delta E_{\pi \pi}}{m_{\pi}}=\frac{4 \pi m_{\mathrm{PS}} a_{0}^{\mathbf{1 4}}}{\left(m_{\mathrm{PS}} L\right)^{3}}\left[1+c_{1} \frac{m_{\mathrm{PS}} a_{0}^{\mathbf{1 4}}}{m_{\mathrm{PS}} L}+c_{2}\left(\frac{m_{\mathrm{PS}} a_{0}^{\mathbf{1 4}}}{m_{\mathrm{PS}} L}\right)^{2}\right],
$$

where $c_{1,2}$ are two known constants, $L$ is the spatial extension of the lattice and $m_{\mathrm{PS}}$ is the Goldstone boson mass.

The interpolating fields for the single and two-particle operator are defined as follows:

$$
\pi^{+}(t) \equiv \sum_{\vec{x}} \bar{d} \gamma_{5} u(\vec{x}, t), \quad\left(\pi^{+} \pi^{+}\right)(t) \equiv \pi^{+}(t+a) \pi^{+}(t)
$$

where $a$ denotes the lattice spacing. Note that the operator for the two-particle state is defined using two single particle states at different time slices in order to avoid issues with Fierz rearrangement [12]. The following two-point functions can then be built:

$$
\begin{aligned}
C_{\pi}(t) & =\left\langle\left(\pi^{+}\right)^{\dagger}\left(t+t_{s}\right) \pi^{+}\left(t_{s}\right)\right\rangle \\
C_{\pi \pi}(t) & =\left\langle\left(\pi^{+} \pi^{+}\right)^{\dagger}\left(t+t_{s}\right)\left(\pi^{+} \pi^{+}\right)\left(t_{s}\right)\right\rangle
\end{aligned}
$$

where $t_{s}$ is the source time slice. The Wick contractions are illustrated in Fig. 1. In order to estimate the necessary correlators, we used stochastic estimators with $Z_{2}$ noise on a single time slice. The
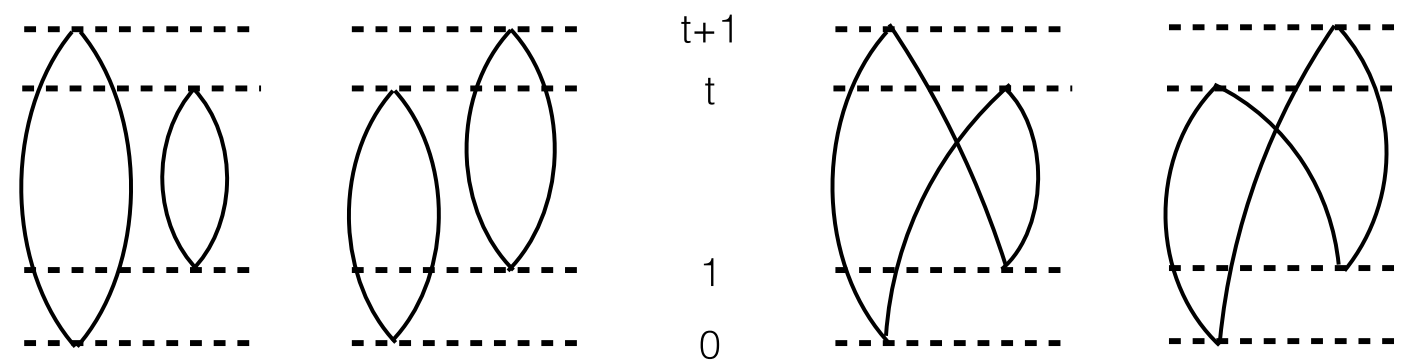

Figure 1: Illustration of the Wick contractions. 
ratio

$$
R(t+a / 2) \equiv \frac{C_{\pi \pi}(t)-C_{\pi \pi}(t+a)}{C_{\pi}^{2}(t)-C_{\pi}^{2}(t+a)}
$$

can be shown to have the following asymptotic behavior for large $t$ :

$$
R(t+a / 2)=A_{R}\left[\cosh \left(\delta E_{\pi \pi t^{\prime}}\right)+\sinh \left(\delta E_{\pi \pi} t^{\prime}\right) \operatorname{coth}\left(2 m_{\mathrm{PS}} t^{\prime}\right)\right]
$$

with $t^{\prime}=t+a / 2-T / 2$. This ratio, designed to remove a constant contribution due the finite extension in time $T$, is sometimes referred as the derivative method [13].

\section{Results}

We show in Fig. 2 the ratio $R$ as a function of time $t / a$ for our various gauge ensembles with different spatial volumes, lattice time extent, lattice spacing and fermion masses. As can be seen the ratio $R(t)$ is determined accurately in all our simulations. We also illustrate a particular choice of fitting range by a blue dotted curve.

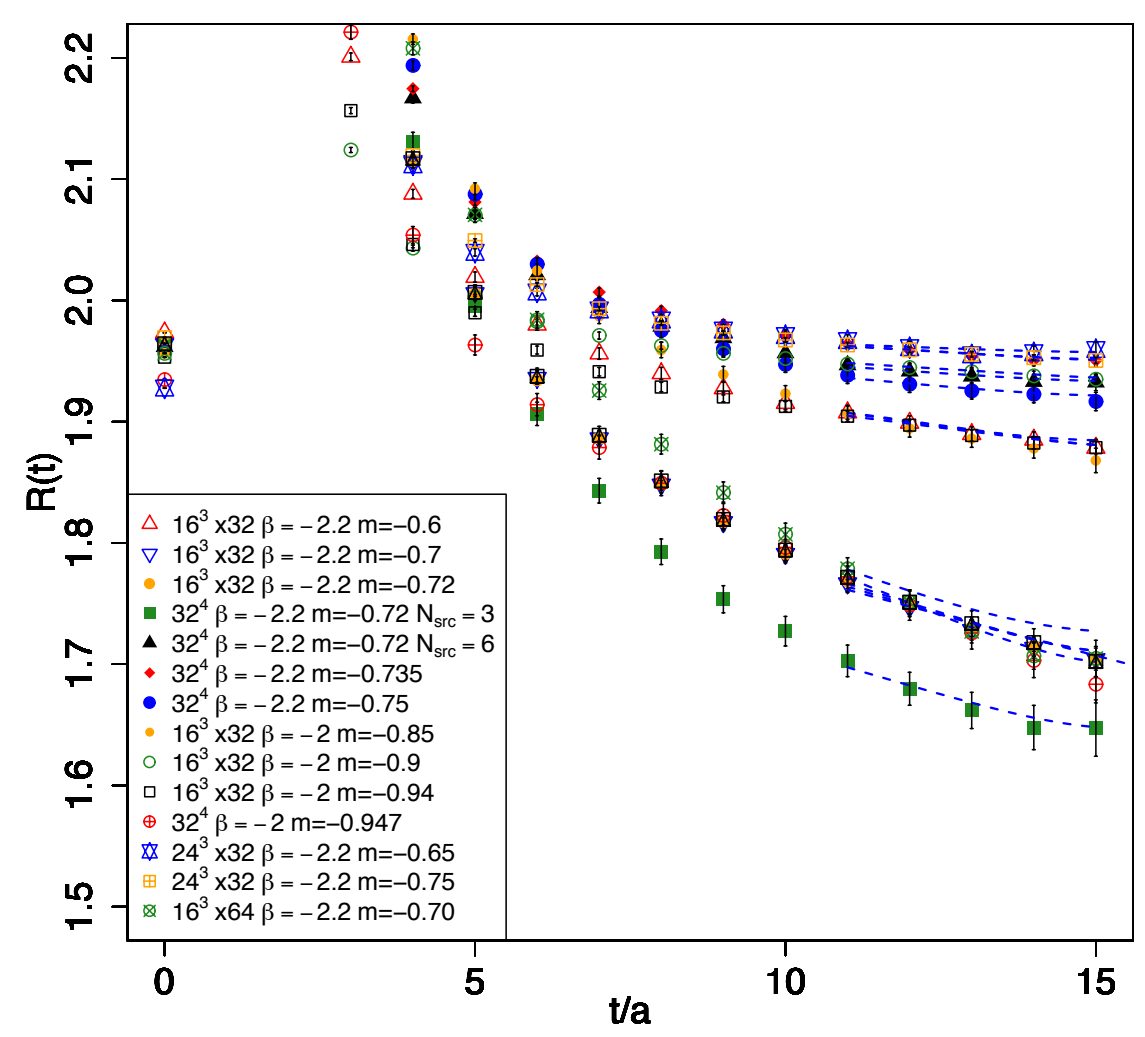

Figure 2: Ratio $R$ defined in Eq. (2.5) as a function of time $t / a$ for our various gauge ensembles with different spatial volumes, lattice time extent, lattice spacing and fermion masses. The blue dotted curves illustrate fits according to Eq. (2.6). 
In order to extract the scattering length $a_{0}^{\mathbf{1 4}} m_{\mathrm{PS}}$ we can then perform a two parameter fit of the correlator on a given range $\left[t_{1}, T / 2\right]$. Using Eq. (2.1) one can then obtain an estimate of $a_{0}^{14} m_{\mathrm{PS}}$. Note that $m_{\mathrm{PS}}$ has been determined in [5] using standard techniques.

In Fig. 3 we show the dependence of the scattering length extracted from the previous figure as a function of the lower bound of the fitting window $t_{1}$. For all the gauge ensembles considered in this work, results show a pronounced dependence indicating a large excited states contamination. This may be due to the use of a local (i.e not smeared) operator. However for $t_{1} \geq 11 a$ one observe that the results do not depend on the fitting range indicating that the asymptotic regime is reached. In what follows, we thus obtain the value of the scattering length choosing $t_{1}=11 \mathrm{a}$ on all our ensembles.

Note that we also checked the convergence of the expansion Eq. (2.1), by looking a posteriori that the relative contribution of the term proportional to $c_{1}$ and $c_{2}$ are small. In practice we observe that the term proportional to $c_{1}$ provides $\sim 10 \%$ of $\delta E_{\pi \pi} / m_{\mathrm{PS}}$ while the term proportional to $c_{2}$ contribute to the order $\sim 1 \%$. It is thus reasonable to assume that Eq. 2.1 is valid and that one does not need to consider higher order corrections in $\frac{m_{\mathrm{PS}} 1_{0}^{14}}{m_{\mathrm{PS}} L}$ for all our ensembles.

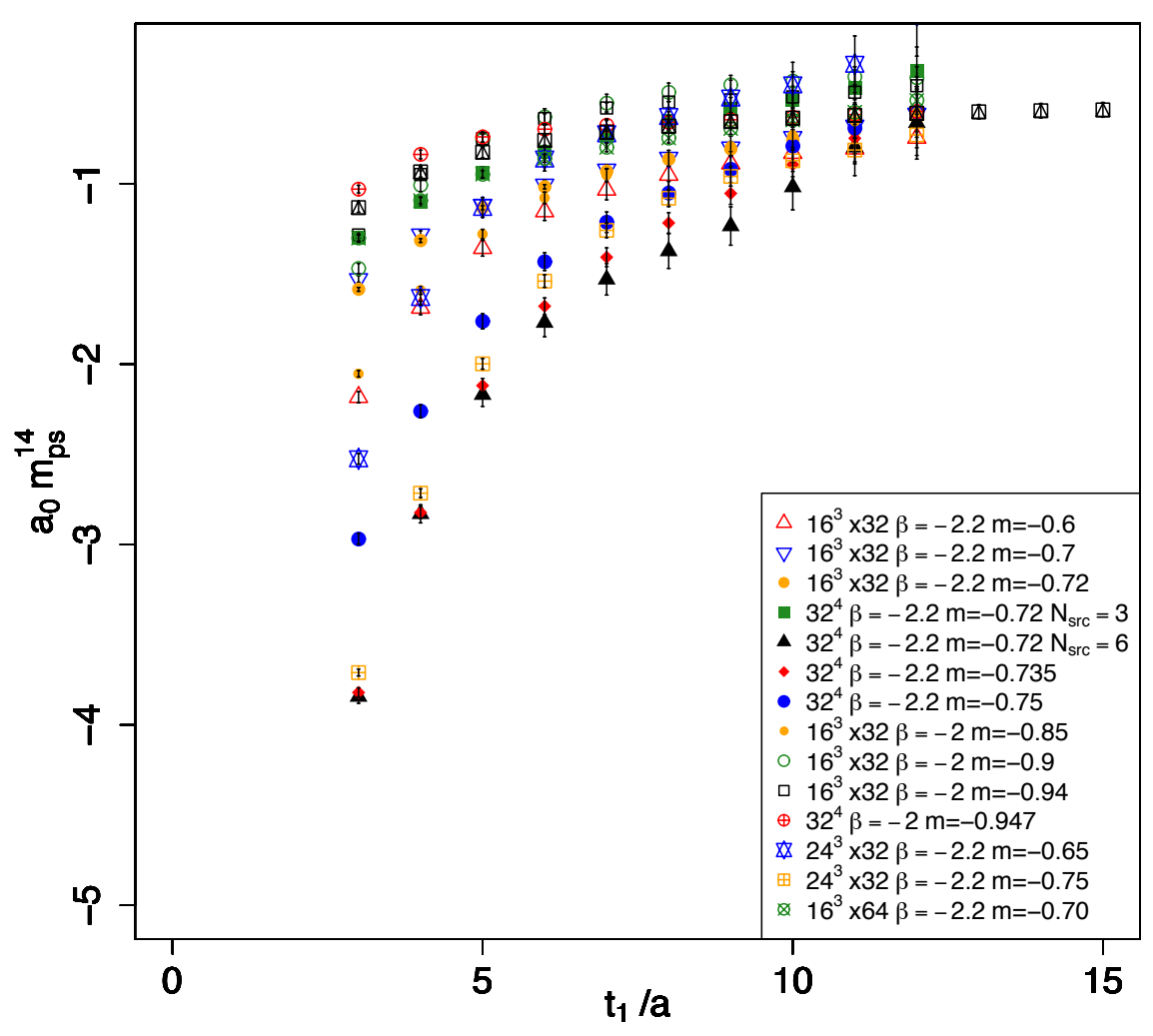

Figure 3: Dependence of the scattering length extracted from the $R(t)$ as a function of the lower bound of the fitting window $t_{1}$. Results show a significant dependence, indicating a large excited states contribution. 
In Fig. 4 we summarize our findings by showing the value of $a_{0}^{14} m_{\mathrm{PS}}$ as a function of the dimensionless ratio $m_{\mathrm{PS}} / f_{\mathrm{PS}}$ for all our ensembles. Note that $f_{\mathrm{PS}}$ is renormalized perturbatively. Conservatively we thus choose a $20 \%$ error bar on the value of the renormalized pseudoscalar decay constant which dominates the horizontal error bar. Vertical error bar are purely statistical and in particular they do not take into account systematic error on the particular choice of a plateau range. However as we argued previously the systematic is most likely to be small compare to our statistical error in view of our precedent discussion. We also show the LO prediction from effective field theory as in Eq. 1.4 [9]. As mentioned in the introduction the LO prediction do not depend on any low energy constants. Within our current statistical and systematic errors the lattice results are compatible with the LO order predictions.

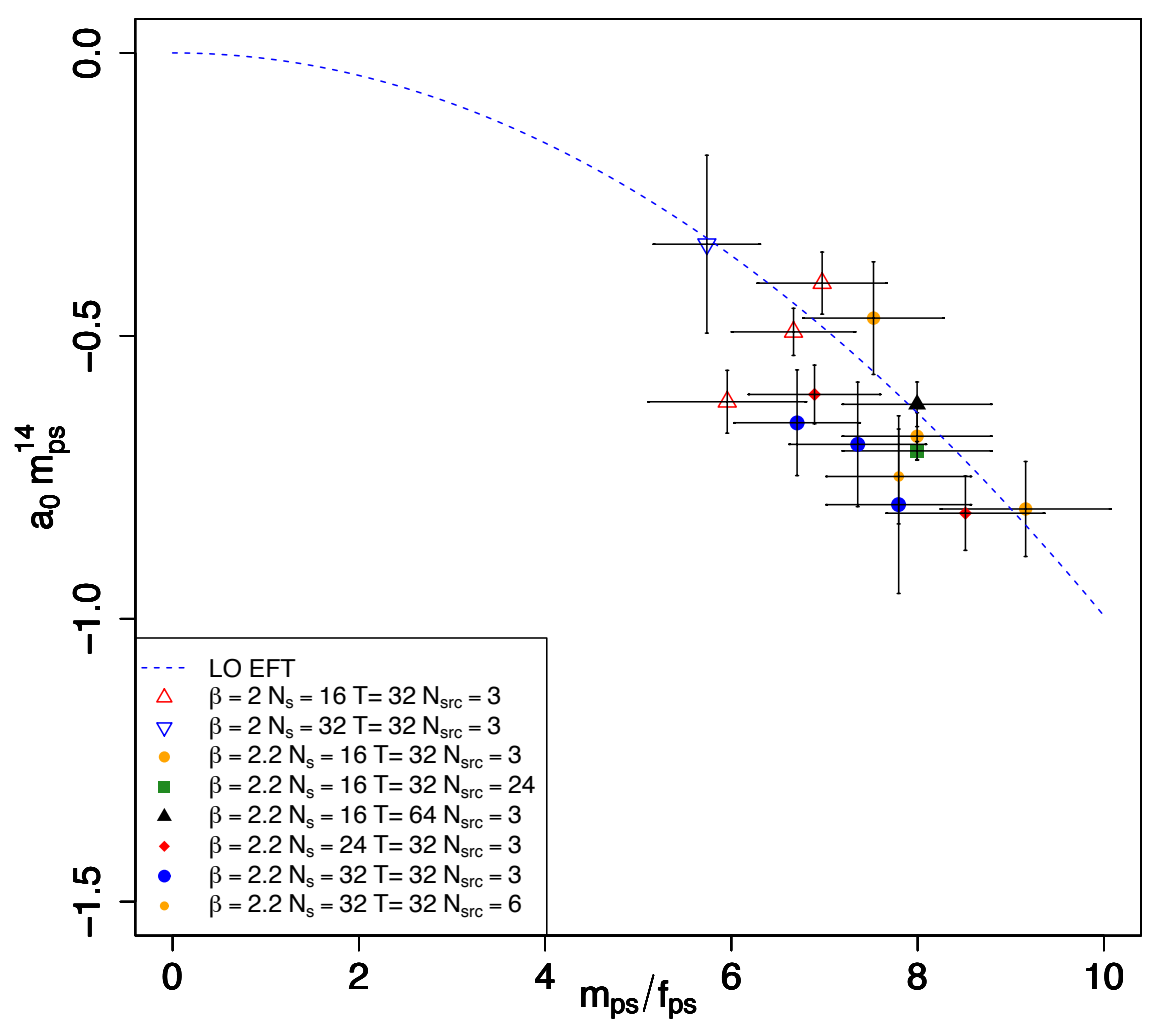

Figure 4: $m_{\mathrm{PS}} a_{0}^{\mathbf{1 4}}$ as a function of the dimensionless ratio $m_{\mathrm{PS}} / f_{\mathrm{PS}}$ for all our ensemble. The vertical error bar are statistical and the horizontal one, comes mostly from the uncertainty on the perturbative value of the renormalization constant of the pseudoscalar meson decay constant. The blue dotted line shows the leading order prediction from Eq. 1.4. The parameters $\beta, N_{S}$ (spatial extent), $T$ (time extent) and $N_{\text {src }}$ (number of stochastic source per configuration) are indicated in the legend. 


\section{Conclusion}

In this study we successfully applied finite size method to the two Goldstone bosons system in an $S U(2)$ gauge theory with two fundamental fermions. We argued that the determination of scattering lengths and more precisely of the corresponding low energy constant can be used to constrain the WW coupling. The result in the particular channel we consider shows good agreement with the leading order prediction from effective field theory. We plan to extend this work to the study of the other channels and in particular to the determination of vector meson decay width which is of fundamental relevance for LHC phenomenology.

\section{Acknowledgments}

This work was supported by the Danish National Research Foundation DNRF:90 grant and by a Lundbeck Foundation Fellowship grant. The computing facilities were provided by the Danish Centre for Scientific Computing.

\section{References}

[1] F. Sannino, Phys. Rev. D 79, 096007 (2009) [arXiv:0902.3494 [hep-ph]].

[2] G. Cacciapaglia and F. Sannino, JHEP 1404, 111 (2014) [arXiv:1402.0233 [hep-ph]].

[3] T. A. Ryttov and F. Sannino, Phys. Rev. D 78, 115010 (2008) [arXiv:0809.0713 [hep-ph]].

[4] T. Appelquist, P. S. Rodrigues da Silva and F. Sannino, Phys. Rev. D 60, 116007 (1999) [hep-ph/9906555].

[5] A. Hietanen, R. Lewis, C. Pica and F. Sannino, JHEP 1407, 116 (2014) [arXiv:1404.2794 [hep-lat]].

[6] R. Lewis, C. Pica and F. Sannino, Phys. Rev. D 85, 014504 (2012) [arXiv:1109.3513 [hep-ph]].

[7] A. Hietanen, et al. these proceedings

[8] B. W. Lee, C. Quigg and H. B. Thacker, Phys. Rev. D 16, 1519 (1977).

[9] J. Bijnens and J. Lu, JHEP 1103, 028 (2011) [arXiv:1102.0172 [hep-ph]].

[10] X. Feng, K. Jansen and D. B. Renner, Phys. Lett. B 684, 268 (2010) [arXiv:0909.3255 [hep-lat]].

[11] M. Luscher, Commun. Math. Phys. 105, 153 (1986).

[12] M. Fukugita, Y. Kuramashi, M. Okawa, H. Mino and A. Ukawa, Phys. Rev. D 52, 3003 (1995) [hep-lat/9501024].

[13] T. Umeda, Phys. Rev. D 75, 094502 (2007) [hep-lat/0701005]. 